Persistence and stability of relative equilibria

Montaldi, James

1997

MIMS EPrint: 2005.37

Manchester Institute for Mathematical Sciences

School of Mathematics

The University of Manchester

\footnotetext{
Reports available from: http://eprints.maths.manchester.ac.uk/

And by contacting: The MIMS Secretary

School of Mathematics

The University of Manchester

Manchester, M13 9PL, UK
} 


\title{
Persistence and Stability of Relative Equilibria
}

\author{
James Montaldi* \\ Institut Non-Linéaire de Nice, \\ 1361 route des Lucioles, 06560 Valbonne, France \\ Final version: September 1996 \\ Published in Nonlinearity 11 (1997), 449-466
}

\begin{abstract}
We consider relative equilibria in symmetric Hamiltonian systems, and their persistence or bifurcation as the momentum is varied. In particular, we extend a classical result about persistence of relative equilibria from values of the momentum map that are regular for the coadjoint action, to arbitrary values, provided that either (i) the relative equilibrium is at a local extremum of the reduced Hamiltonian, or (ii) the action on the phase space is (locally) free. The first case uses just point-set topology, while in the second we rely on the local normal form for (free) symplectic group actions, and then apply the splitting lemma. We also consider the Lyapunov stability of extremal relative equilibria. The group of symmetries is assumed to be compact.
\end{abstract}

\section{Introduction}

A relative equilibrium in a symmetric dynamical system is a group orbit that is invariant under the dynamics. Another way of viewing a relative equilibrium is to consider the dynamics induced on the orbit space of the phase space, and then a relative equilibrium is just an equilibrium point of this induced dynamics. For finite groups, relative equilibria are just (group orbits of) equilibria; in this paper we are therefore only interested in continuous symmetries.

In a symmetric Hamiltonian system there is the added structure of a momentum map, whose level sets are invariant under the dynamics ("conservation of momentum"). The 'persistence question' we address is whether, given a relative equilibrium on the $\alpha$-level set of the momentum map, we can deduce the existence of relative equilibria on nearby level sets.

There is a well-known observation due to Arnold, that if there is a non-degenerate relative equilibrium for a given value $\alpha$ of the momentum map, where $\alpha$ satisfies a certain condition of regularity, then on each level set of the momentum map close to the $\alpha$-level set, there is a unique non-degenerate relative equilibrium in a neighbourhood of the given one (the relative equilibrium persists). The regularity hypothesis is that $\alpha$ be a regular point for the coadjoint action of the Lie group. The non-degeneracy of the relative equilibrium requires firstly that the points of the relative equilibrium have trivial isotropy (or more generally that the orbit space be smooth) and secondly, that the reduced Hamiltonian $H_{\alpha}$ have a non-degenerate critical point at the relative equilibrium. The proof is a straightforward application of the implicit function theorem on the smooth orbit space. The observation continues by pointing out

* research partially supported by the Alliance programme for scientific cooperation between Great Britain and France. AMS 1991 classification: 58F05, 70H33, 58F14 
that if the quadratic part of the reduced Hamiltonian $H_{\alpha}$ is positive definite, then the relative equilibrium is Lyapunov stable (even to perturbations changing the momentum value) [2,9]. This approach to proving stability of a relative equilibrium is called the Energy-Casimir method, in contrast with the closely related Energy-Momentum method developed by Marsden and co-workers. For more details and further references, see Marsden's lecture notes [11].

An example in [9] (Exercise 15.10 of Chapter IV) shows that in general the regularity of $\alpha$ is necessary. The group in that example is the group of affine transformations of the real line, which is not compact. In this paper we consider the case where the group is compact, and show that one may draw the same conclusions of persistence and stability even without the regularity hypotheses, provided the relative equilibrium is extremal; that is, the reduced Hamiltonian $H_{\alpha}$ has a local extremum at the relative equilibrium (Theorem 1.2). We also give an example in $\S 1$ to show that persistence may fail for non-extremal relative equilibria.

George Patrick [18] has shown that in a neighbourhood of a relative equilibrium where the action is locally free and the velocity $\xi$ is regular, the set of relative equilibria forms a smooth submanifold of phase space (of dimension $\operatorname{dim} G+\operatorname{rank} G$ ), which implies the persistence result in this case whether or not the relative equilibrium is extremal, and whether or not $\alpha$ is regular in $\mathfrak{g}^{*}$ (see Remark 3.3(e)).

The remainder of the paper is dedicated to a better understanding of the case where the group acts freely (or locally freely - see Remark 3.6), but the value $\alpha$ of the momentum map is not regular for the coadjoint action. Of particular interest is the case where the velocity of the relative equilibrium is a regular element of the Lie algebra, since the results are particularly clean. We now briefly describe these later results.

Throughout this paper, we suppose that $G$ is a compact Lie group acting on a symplectic manifold $(P, \omega)$ in such a way that there exists an equivariant momentum map $\Phi: P \rightarrow \mathfrak{g}^{*}$. We will also suppose that $H: P \rightarrow \mathbf{R}$ is a smooth $G$-invariant function (the Hamiltonian).

Let $\mathfrak{t}$ be a Cartan subalgebra of $\mathfrak{g}$ (the Lie algebra of a maximal torus of $G$ ), and $\mathfrak{t}^{*}$ its dual. Recall that any element of $\mathfrak{g}$ is conjugate to an element of $\mathfrak{t}$; that is, every adjoint orbit in $\mathfrak{g}$ intersects $\mathfrak{t}$. Let $W$ be the Weyl group of $G$, which acts both on $\mathfrak{t}$ and on $\mathfrak{t}^{*}$. For $\mu \in \mathfrak{t}^{*}$, let $w(\mu)$ be the cardinality of the W-orbit in $\mathrm{t}^{*}$ containing $\mu$.

The second principal result of the paper (Theorem 3.1) is the following. Suppose that $\bar{p} \in P / G$ is a non-degenerate relative equilibrium with momentum $\alpha$ (for a given invariant Hamiltonian), and $p$ has trivial isotropy, so that the group acts freely in a neighbourhood of $\bar{p}=$ G.p. Then for all $P_{\mu}$ with $\mu$ sufficiently close to $\alpha$, there are at least $w(\mu) / w(\alpha)$ relative equilibria on $P_{\mu}$, near $\bar{p}$, provided they are all non-degenerate. If moreover, the velocity of the relative equilibrium (defined modulo the adjoint action) is regular in $\mathfrak{g}$, we say that the relative equilibrium is regular, and in that case, there are precisely $w(\mu) / w(\alpha)$ relative equilibria on $P_{\mu}$, near $\bar{p}$, which are all non-degenerate, see Theorem 3.2. One can also make a similar (though numerically weaker) conclusion if the action is only locally free, see Remark 3.6. At the end of the paper we consider briefly the eigenvalues of the relative equilibria on $P_{\mu}$ near a regular relative equilibrium on $P_{\alpha}$, showing that generically they are all imaginary provided the relative equilibrium on $P_{\alpha}$ is strongly stable.

As an example, consider the case where the group is $\mathbf{S O}(3)$. A relative equilibrium with trivial isotropy is regular if and only if it is not an orbit of equilibria. If $\alpha=0$ for such a regular relative equilibrium, then for $\mu \neq 0$ (but sufficiently small) there are precisely $2(=w(\mu))$ relative equilibria on $P_{\mu}$ near the given one (see Example 3.4). On the other hand in the rigid body problem, the zero momentum set contains (is) a relative equilibrium with trivial isotropy, where the velocity is zero. For a fixed non-zero value of the momentum, there are 6 relative equilibria, corresponding to rotation in each direction about each of the principal axes of inertia. Such non-regular relative equilibria are dealt with in more detail in a later paper [14]. 
In [20], Roberts and Sousa-Dias describe a complementary approach to the question of bifurcations of relative equilibria.

\section{Notation and context}

Throughout, we assume that $G$ is a compact Lie group. Its Lie algebra is denoted $\mathfrak{g}$, and the dual of its Lie algebra by $\mathfrak{g}^{*}$.

$P$ is a finite dimensional symplectic manifold with symplectic form $\omega$. The Lie group $G$ acts on $P$ symplectically, and for $p \in P$ we write $\bar{p}=G$. $p$ for the orbit through $p$, considered either as a subset of $P$ or as an element of the orbit space $P / G$. The tangent space to the orbit $\bar{p}$ at $p$ is denoted $\mathfrak{g} . p$. The isotropy group for the point $p$ is denoted $G_{p}$, and its Lie algebra by $\mathfrak{g}_{p}$.

For $\xi \in \mathfrak{g}$, the associated vector field on $P$ is denoted $X_{\xi}$, and given a Hamiltonian function $H$ : $P \rightarrow \mathbf{R}$, the associated vector field is denoted $X_{H}$.

There are natural actions of $G$ on $\mathfrak{g}$ and $\mathfrak{g}^{*}$ — the adjoint and coadjoint actions respectively. For $\mu \in \mathfrak{g}^{*}$ we write $O_{\mu}$ for the coadjoint orbit through $\mu$ and $\mathfrak{g} . \mu$ for its tangent space at $\mu$.

We assume that the symplectic action is such that there exists an equivariant momentum map,

$$
\Phi: P \longrightarrow \mathfrak{g}^{*}
$$

The equivariance is with respect to the given action on $P$ and the coadjoint action on $\mathfrak{g}^{*}$ :

$$
\Phi(g \cdot p)=A d_{g^{-1}}^{*} \Phi(p) \text {. }
$$

For details about when such a momentum map exists the reader should consult [7] or [9]. Recall that the momentum map is defined by the differential condition,

$$
\left\langle d \Phi_{p}(v), \xi\right\rangle=\omega\left(X_{\xi}, v\right), \quad \forall \xi \in \mathfrak{g}, \forall v \in T_{p} P
$$

Note that if a (non-equivariant) momentum map $\Phi: P \rightarrow \mathfrak{g}^{*}$ exists, then the map $\Phi^{g}: P \rightarrow \mathfrak{g}^{*}$ defined by

$$
\Phi^{g}(p)=A d_{g^{-1}}^{*} \Phi\left(g^{-1} \cdot p\right)
$$

is also a momentum map for the given action. As the group $G$ is compact we can average $\Phi^{g}$ over the group to obtain an equivariant momentum map.

The coadjoint action on $\mathfrak{g}^{*}$ has a quotient which is a smooth manifold with boundary and corners (it can be naturally identified with a Weyl chamber in $\mathfrak{t}^{*}$ ). Since $\Phi$ is assumed to be equivariant, the image of an orbit G.p in $P$ is an orbit $O_{\alpha}$ in $\mathfrak{g}^{*}$, where $\alpha=\Phi(p)$. Thus $\Phi$ passes down to a map $\varphi$ of the orbit spaces, called the orbit momentum map, in such a way that the following diagram commutes:

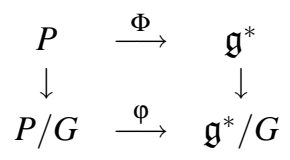

The components of the map $\varphi$ are Casimirs for the natural Poisson structure on the orbit space.

The Meyer-Marsden-Weinstein reduced spaces [13, 12] are defined to be

$$
P_{\mu}=\frac{\Phi^{-1}(\mu)}{G_{\mu}}=\frac{\Phi^{-1}\left(O_{\mu}\right)}{G} .
$$


The equivalence of the two definitions as sets is obvious, while as analytic spaces (with their rings of functions) it is less obvious; it follows from the slice theorem, and so requires the compactness of the group. The second definition has the advantage that it realizes the reduced spaces as subspaces of the orbit space $P / G$, and in particular $\Phi^{-1}\left(O_{\mu}\right) / G=\varphi^{-1}\left(O_{\mu}\right)$. Thus we can take the definition of the reduced spaces to be

$$
P_{\mu}:=\varphi^{-1}\left(O_{\mu}\right)
$$

Note that if $v \in O_{\mu}$ then $O_{\mu}=O_{\nu}$ and $P_{\mu}=P_{\nu}$.

If $H: P \rightarrow \mathbf{R}$ is an invariant Hamiltonian function, it passes down to a function $\bar{H}: P / G \rightarrow \mathbf{R}$, whose restriction to the reduced space $P_{\mu}$ is denoted $H_{\mu}$. If $P_{\mu}$ is smooth, a relative equilibium $p_{\mu} \in P_{\mu}$ is non-degenerate if $d^{2} H_{\mu}\left(p_{\mu}\right)$ is a non-degenerate quadratic form; it is regular if the velocity $\xi$ of the relative equilibrium is a regular element of $\mathfrak{g}$. (The velocity is defined by $X_{H}(p)=X_{\xi}(p)$ for some point $p$ of the relative equilibrium; this velocity is well-defined modulo the adjoint action, since by equivariance $X_{H}(g \cdot p)=X_{\operatorname{Ad}_{g}(\xi)}(g \cdot p)$.)

For simplicity, we assume throughout that the Hamiltonian $H$ is such that the associated vector field $X_{H}$ is complete.

\section{Persistence of extremal relative equilibria}

A relative equilibrium of a symmetric Hamiltonian system is a group orbit that is invariant under the dynamics. It is thus a point of the orbit space $P / G$ that is invariant under the dynamics induced thereon - that is, an equilibrium point on $P / G$. Any reduced space $P_{\alpha}$ has the structure of a stratified symplectic space [21], and relative equilibria occur at points on the strata where the restriction of the Hamiltonian $H_{\alpha}$ to that stratum has a critical point. In this section we are interested in the situation where $p$ is a local extremum of $H_{\alpha}$ on $P_{\alpha}$. Since the restriction to the symplectic stratum containing $p$ will also have a local extremum at $p$, such a point is clearly a relative equilibrium. We show that extremal relative equilibria are Lyapunov stable, and that they persist to nearby reduced spaces (to $P_{\mu}$ for $\mu \approx \alpha$ ).

Definition A relative equilibrium $p \in P_{\alpha}$ is said to be extremal if $H_{\alpha}$ has a local extremum at $p$.

Note that if $p$ is an extremal relative equilibrium with $H_{\alpha}(p)=h$, then $p$ is an isolated point of $H_{\alpha}^{-1}(h)$.

Example 1.1 We give a simple example illustrating the notion of extremal relative equilibrium, and showing why in general it is a necessary condition for both persistence and stability.

Consider the phase space $P=\mathbf{R}^{3} \times \mathbf{R}^{3}$, with coordinates $q_{i}, p_{i}(i=1,2,3)$, and canonical symplectic structure $\omega=d q_{1} \wedge d p_{1}+d q_{2} \wedge d p_{2}+d q_{3} \wedge d p_{3}$. We take as group $\mathbf{S O}(3)$ acting diagonally by rotations:

$$
R \mapsto\left(\begin{array}{cc}
R & 0 \\
0 & R
\end{array}\right) \quad \in \mathbf{S} \mathbf{p}\left(\mathbf{R}^{3} \times \mathbf{R}^{3}\right)
$$

where $R \in \mathbf{S O}(3)$. Note that the isotropy subgroup for the origin is $\mathbf{S O}(3)$, while for any other point it is either SO(2) if $q$ and $p$ are parallel, and trivial otherwise. The invariants of this action are functions of the following 3 fundamental invariants,

$$
\begin{aligned}
& A=|p|^{2} \\
& B=|q|^{2} \\
& C=q \cdot p=q_{1} p_{1}+q_{2} p_{2}+q_{3} p_{3}
\end{aligned}
$$


The orbit space can be embedded as the subset of $\mathbf{R}^{3}$ given by the conditions $A, B \geq 0$ and $C^{2} \leq A B$, a solid cone. The momentum map is the angular momentum,

$$
\Phi\left(q_{1}, q_{2}, q_{3}, p_{1}, p_{2}, p_{3}\right)=q \wedge p .
$$

The orbit momentum map $\varphi: P / G \rightarrow \mathfrak{g}^{*} / G$ is given by

$$
\varphi(A, B, C)=A B-C^{2} .
$$

The reduced space $P_{0}$ is the half-cone $A B=C^{2}, A \geq 0$, which has two strata: the origin and the rest. The other reduced spaces $P_{\mu}$ are each one sheet of a two-sheeted hyperboloid (see Fig. 1).

Consider the Hamiltonian function $H=A-B$. The origin is a relative equilibrium which is not extremal, and it is easy to see from the figure that it is the only relative equilibrium - indeed, every solution except for the origin is unbounded. On the other hand, if we take $H=A+B$, then $H_{0}=$ $(A+B)_{P_{0}}$ has a strict minimum at the origin, so that in this case the origin is an extremal relative equilibrium and here we get a single relative equilibrium on each reduced space; Theorem 1.2 below predicts at least one on each reduced space. Had the action been free, Theorem 3.1 would have predicted 2 relative equilibria on each reduced space, since the Weyl group of $\mathbf{S O}(3)$ is $\mathbf{Z} / \mathbf{2 Z}$.

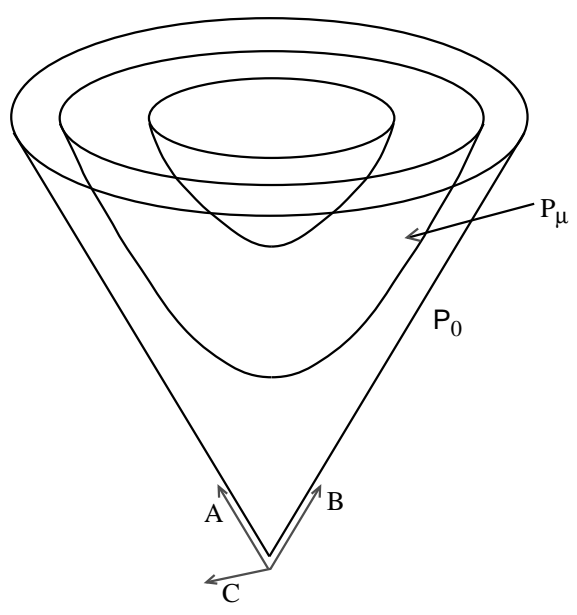

Figure 1: The reduced spaces are each one sheet of a 2-sheeted hyperboloid

Suppose $\bar{p} \in P_{\alpha}$ is a relative equilibrium; it is well-known (e.g. Appendix 2 of [2], or Chapter IV, Section 8 of [9]) that if, (i) the action on $P$ is simple (i.e. the orbit space has the structure of a differentiable manifold), (ii) $\alpha$ is a regular point of the coadjoint action of $G$, (iii) $P_{\alpha}$ is non-singular at $p$, and (iv) $d^{2} H_{\alpha}(p)$ is definite, then the group orbit $G . p$ in $P$ is (relatively) Lyapunov stable for the dynamics associated to $H$. Moreover, on nearby reduced spaces there is also a relative equilibrium. The proof is a straightforward application of the implicit function theorem.

The purpose of this section is to prove essentially the same result while dropping the regularity conditions, but adding a hypothesis on the compactness of the group. In particular, we show that if $p$ is isolated in $H_{\alpha}^{-1}(h)$ (where $h=H_{\alpha}(p)$ ) then the corresponding group orbit in $P$ is relatively Lyapunov stable. The proof uses only elementary point-set topology. 
With the same hypothesis we also prove a persistence result, showing that on each reduced space near the given one, there is at least one relative equilibrium.

Given a Hamiltonian $H$, there is an associated flow

$$
\sigma: P \times \mathbf{R} \rightarrow P
$$

it is of course $G$-equivariant. This flow on $P$ descends to a flow on the orbit space

$$
\bar{\sigma}: P / G \times \mathbf{R} \rightarrow P / G
$$

Since $G$ is compact, $P / G$ is naturally a Poisson space, and the flow can be defined directly in terms of the "quotient Hamiltonian" $\bar{H}: P / G \rightarrow \mathbf{R}$ and the Poisson structure.

A relative equilibrium is an equilibrium point of the flow $\bar{\sigma}$ on $P / G$. Recall that a compact invariant set $S \subset P$ (or in $P / G$ ) is Lyapunov stable if for any neighbourhood $U$ of $S$, there exists a neighbourhood $V$ of $S$ in $P$ (respectively in $P / G$ ) with $\sigma(V \times \mathbf{R}) \subset U$. There is usually a distinction made between Lyapunov stability and relative Lyapunov stability in $P$, where the neighbourhoods are required to be $G$-invariant. However, if $S$ is $G$-invariant, with $G$ compact, then these notions are equivalent, because any neighbourhood of $S$ contains a $G$-invariant neighbourhood. For a dynamical interpretation of the stability of relative equilibria see $[17,18]$.

Theorem 1.2 Suppose $\bar{p} \in P_{\alpha} \subset P / G$ is an extremal relative equilibrium. Then

(i) $\bar{p}$ is Lyapunov stable in P/G. Consequently G.p $=\pi^{-1}(\bar{p})$ is Lyapunov stable in P;

(ii) for all $\mu$ close to $\alpha$ in the image under $\varphi$ of a neighbourhood of $\bar{p}$, there is a relative equilibrium on $P_{\mu}$ close to $\bar{p}$.

Remarks 1.3 (a) If $\bar{p}$ is not a strict local extremum, but belongs to a compact set $S \subset P_{\alpha}$ on which $H_{\alpha}$ takes its extremal value (in which case every point of $S$ is a relative equilibrium), then $\bar{p}$ may not be Lyapunov stable, but one shows in exactly the same manner that $S$ is. Moreover, part (ii) also holds, after replacing $\bar{p}$ by $S$.

(b) In the conclusion of (ii), the relative equilibrium is not necessarily isolated. If it is isolated it is Lyapunov stable by (i). In general, the compact set of relative equilibria will be Lyapunov stable by (a). Such a situation occurs in the symmetric rigid body problem, that has $\mathbf{S O}(3) \times \mathbf{S O}(2)$ symmetry. If we just quotient by $\mathbf{S O}(3)$ (as for the non-symmetric rigid body) then on each reduced space for non-zero momentum, there is a circle of relative equilibria.

(c) Note that the proof of (ii) also implies that extremal relative equilibria persist under small perturbations of the Hamiltonian.

(d) In fact compactness of the group is not really needed for part (i), since the crucial point (see the Lemma below) is only that the quotient topology on the orbit space $\mathfrak{g}^{*} / G$ is Hausdorff, a condition which fails for the example of Libermann and Marle referred to above, but which is verified trivially for any Abelian group.

(e) Part (i) can also be seen as a result about stability of Poisson-Hamilton equilibria, in the sense of Weinstein [22]. In his paper, Weinstein points out that care should be taken in deducing Lyapunov stability of Poisson-Hamilton equilibria from knowledge of the second variation of the Hamiltonian on the symplectic leaf containing the equilibrium. The Poisson spaces that arise as the orbit space of a symplectic action of a compact group form a restricted class, and the lesson from this paper is that even for this restricted class it is certainly not sufficient to consider the restriction of the Hamiltonian to the symplectic leaf: one must consider the entire reduced space, so in particular one must also 
consider those symplectic leaves containing the given one in their closure. However, for more general Poisson spaces even this is not sufficient, as the planar pendulum example of Weinstein on p.8 of [22] shows.

The proof of the theorem is just point-set topology. The first part relies on the following lemma. Recall that a precompact set is a set with compact closure, and a precompact neighbourhood is open.

Lemma 1.4 Let $f: X \rightarrow Y$ be a continuous map, with $X$ locally compact and $Y$ Hausdorff. Suppose $y \in Y$ is such that $S=f^{-1}(y)$ is compact. Then for any precompact neighbourhood $U$ of $S$ there exists a neighbourhood $V$ of $y$ such that $f^{-1}(V)$ and $\partial U$ are disjoint.

PROOF First note that since $X$ is locally compact, $S$ does indeed have a precompact neighbourhood: each point of $S$ has a precompact neighbourhood, so extracting a finite subcover we obtain the desired precompact neighbourhood of $S$. Let $V_{\beta}$ be a fundamental system of closed neighbourhoods of $y \in Y$ (that is, $\cap_{\beta} V_{\beta}=\{y\}$ ), and let $Z_{\beta}=f^{-1}\left(V_{\beta}\right)$, which is closed. Then $S=\cap Z_{\beta}$, and since $S \cap \partial U=\emptyset$, we have $\partial U \subset \cup Z_{\beta}^{\prime}$ (the complements of the $Z_{\beta}$ ). Now, $\partial U$ is compact, so we can extract a finite subcover $\partial U \subset Z_{\beta_{1}}^{\prime} \cup \ldots \cup Z_{\beta_{n}}^{\prime}$. Then

$$
V=\bigcap_{i=1}^{n} V_{\beta_{i}}^{\mathrm{int}}
$$

is a neighbourhood of $y$ with the required property.

Proof (of theorem) (i) We apply the lemma with $f=(\varphi, H)$ and $X=P / G$, where $\varphi$ is the orbit momentum map. Let $U$ be a precompact neighbourhood of $S=f^{-1}(\mu, h)$, and let $V$ be a neighbourhood of $(\mu, h)$ satisfying $f^{-1}(V) \cap \partial U=\emptyset$. Then $f^{-1}(V) \cap U$ is a flow-invariant neighbourhood of $S$ as required, since $f^{-1}(V)$ is flow-invariant, and flow preserves connected components.

(ii) Suppose that $\bar{p}$ is a local minimum of $H_{\alpha}$ (the case of a local maximum is analogous). Let $K$ be a compact neighbourhood of $\bar{p}$, small enough that on $K \cap P_{\alpha}$, we have $\bar{H}^{-1}(0)=\bar{p}$, and such that $K$ is the closure of its interior. For each $\mu \in \varphi(K)$, put $K_{\mu}=K \cap P_{\mu}$.

For each $\mu$ we have that $H_{\mu}\left(K_{\mu}\right)$ is compact; let $\bar{p}_{\mu} \in K_{\mu}$ be a point that realizes the minimum of $\left.H\right|_{K_{\mu}}:$

$$
H_{\mu}\left(\bar{p}_{\mu}\right)=\inf \left\{H(x) \mid x \in K_{\mu}\right\} .
$$

Note that if $\bar{p}_{\mu} \in K^{o}$ (interior of $K$ ) we are done, for then $\bar{p}_{\mu}$ is a local minimum.

It remains to show that if $\mu$ is sufficiently close to $\alpha$, then $\bar{p}_{\mu} \in K^{o}$. If this were not so, then by compactness of $K$, the family $\bar{p}_{\mu}$ would have a convergent subsequence (as $\mu \rightarrow 0$ ) with $\bar{p}_{\mu_{i}} \rightarrow q \in K_{\alpha}$, with $q \neq \bar{p}$. But then $\bar{H}\left(\bar{p}_{\mu_{i}}\right) \rightarrow \bar{H}(q)>\bar{H}(\bar{p})$, which is absurd.

\section{Local Model}

In this section, we describe a model for the symplectic action of $G$ on $P$ in a neighbourhood of the orbit G.p. This is essentially due to Guillemin and Sternberg [7] and Marle [10], see also [21]. We give what we feel to be a simpler presentation, using the standard slice theorem together with the equivariant Darboux-Weinstein theorem [7], rather than the isotropic embedding theorem.

For the ordinary (non-symplectic) case, a local model for the action of a compact Lie group is provided by the slice theorem. Let $S_{p}:=T_{p} P / \mathfrak{g} . p$. As the isotropy group $G_{p}$ is compact, and the group orbit is $G_{p}$-invariant, it follows that we can (and do) identify a neighbourhood of 0 in $S_{p}$ with a $G_{p}$-invariant submanifold of $P$ through $p$, transverse to the group orbit. 
The slice theorem [4] states that there is an equivariant diffeomorphism

$$
G \times_{G_{p}} S_{p} \stackrel{\sim}{\longrightarrow} P
$$

where the $G$-action on the model space $G \times{ }_{G_{p}} S_{p}$ is given by $g$. $[h, s]=[g h, s]$. The diffeomorphism is simply given by $[h, s] \mapsto h \cdot s$. The image of this diffeomorphism is an invariant neighbourhood of the orbit G.p in $P$.

Note that when considering only free actions in the next section, this local model is even simpler: $G \times S_{p} \stackrel{\sim}{\longrightarrow} P$.

In the symplectic case, we need to include the symplectic form, which refines the decomposition $T_{p} P=\mathfrak{g} \cdot p \oplus T_{p} S_{p}$, and in addition it is useful to have an expression for the momentum map in the local model.

We define four vector spaces as follows (for details see [15] or [1]):

$$
\begin{aligned}
W_{p} & =\operatorname{ker} d \Phi_{p} \cap \mathfrak{g} \cdot p=\mathfrak{g}_{\alpha} \cdot p \\
X_{p} & =\mathfrak{g} \cdot p / W_{p} \\
Y_{p} & =\operatorname{ker} d \Phi_{p} / W_{p} \\
Z_{p} & =T_{p} P /\left(\mathfrak{g} \cdot p+\operatorname{ker} d \Phi_{p}\right) .
\end{aligned}
$$

Here $\alpha=\Phi(p)$. (In [1] it is assumed that $\alpha=0$, so $X_{p}=0$.) Since the group is compact, these spaces can be identified with $G_{p}$-invariant submanifolds of $P$. There are natural isomorphisms,

$$
\begin{aligned}
W_{p} & \cong \mathfrak{g}_{\alpha} / \mathfrak{g}_{p} \\
X_{p} & \cong \mathfrak{g} / \mathfrak{g}_{\alpha} \\
Z_{p} & \cong\left(\mathfrak{g}_{\alpha} / \mathfrak{g}_{p}\right)^{*}
\end{aligned}
$$

The spaces $X_{p}$ and $Y_{p}$ are symplectic, while $W_{p}$ is isotropic and dually paired with $Z_{p}$ by the symplectic form, whence the isomorphism for $Z_{p}$ above. The symplectic form on $X_{p}$ is isomorphic to the KostantKirillov symplectic form on $\mathfrak{g} / \mathfrak{g}_{\alpha}$. (Note that as $G_{p}$ is compact, there is a $G_{p}$-equivariant almostcomplex structure $J_{p}$ on $T_{p} P$, compatible with the symplectic form; if we choose $Z_{p}=J\left(W_{p}\right)$ then $Z_{p}$ is also isotropic.)

The space $Y_{p}$ is called the symplectic slice at $p$ :

$$
Y_{p}=\frac{\operatorname{ker} d \Phi_{p}}{\mathfrak{g}_{\alpha} \cdot p} ;
$$

it plays the role in symplectic geometry of the ordinary slice. Being a symplectic space with a $G_{p^{-}}$ action, it has its own momentum map,

$$
\Phi_{Y_{p}}: Y_{p} \rightarrow \mathfrak{g}_{p}^{*}
$$

Since $G$ is compact, there is a $G$-invariant scalar product on $\mathfrak{g}^{*}$. Following [21], we can use this to define a splitting $\mathfrak{g}_{\alpha}=\mathfrak{g}_{p} \oplus \mathfrak{m}$, and correspondingly

$$
\mathfrak{g}_{\alpha}^{*}=\mathfrak{g}_{p}^{*} \oplus \mathfrak{m}^{*}
$$

The subspace $\mathfrak{m}^{*}$ can be identified naturally with the annihilator of $\mathfrak{g}_{p}$ in $\mathfrak{g}_{\alpha}^{*}$. (Note that $\mathfrak{m}$ is not in general a Lie subalgebra of $\mathfrak{g}_{\alpha}$.) 
Proposition 2.1 (Local Model) Let $p \in P$ be such that $\Phi(p)=\alpha$. Then there is a neighbourhood of $G . p$, and a G-equivariant isomorphism of symplectic manifolds

$$
G \times{ }_{G_{p}}\left(\mathfrak{m}^{*} \times Y_{p}\right) \stackrel{\sim}{\longrightarrow} P .
$$

The momentum map for the G-action on the model space is given by,

$$
\Phi([g, v, v])=\operatorname{Ad}_{g^{-1}}^{*}\left(v \oplus \Phi_{Y_{p}}(v)\right) .
$$

Note that $\boldsymbol{v} \in \mathfrak{m}^{*}$ and $\Phi_{Y_{p}}(v) \in \mathfrak{g}_{p}^{*}$, so that under the identification above, $\left(\boldsymbol{v} \oplus \Phi_{Y_{p}}(v)\right) \in \mathfrak{g}^{*}$.

Proof The equivariant Darboux-Weinstein theorem [7] states that if two symplectic spaces are $G$-equivariantly diffeomorphic, and such that the diffeomorphism identifies the symplectic structures at each point of a pair of $G$-invariant submanifolds, then the diffeomorphism can be made (via an equivariant homotopy) into a $G$-symplectomorphism between neighbourhoods of the two submanifolds. This is precisely the situation we have here: the diffeomorphism is given by the classical slice theorem, and the two submanifolds are the group orbits $G . p$ and $G \times_{G_{p}}\{0\}$. The slice theorem identifies the symplectic forms at corresponding points of the two orbits, since they have identical $W, X, Y, Z$-decompositions.

Corollary 2.2 Let $\bar{p}=G . p \in P / G$. In a neighbourhood of $\bar{p}$, the orbit space is isomorphic as a Poisson space to,

$$
\left(\mathfrak{m}^{*} \times Y_{p}\right) / G_{p} \stackrel{\sim}{\longrightarrow} P / G
$$

(for some Poisson structure on this model orbit space) and the orbit momentum map $\varphi:\left(\mathfrak{m}^{*} \times\right.$ $\left.Y_{p}\right) / G_{p} \rightarrow \mathfrak{g}^{*} / G$ becomes

$$
\varphi([v, v])=G \cdot\left(v \oplus \Phi_{Y_{p}}(v)\right) .
$$

(In this paper, we do not make explicit use of the Poisson structure on $\left(\mathfrak{m}^{*} \times Y_{p}\right) / G_{p}$.)

In $\S 4$, we will need to identify the differential of an invariant Hamiltonian function with an element of $\mathfrak{g}_{\mu} / \mathfrak{g}_{p}$; this is done as follows. Note that if $H$ is a $G$-invariant Hamiltonian on $P$, then the differential $d H_{p}$ at $p$ annihilates $W_{p} \oplus X_{p}$. Furthermore, if $p$ is a relative equilibrium of $H$, then $d H_{p}$ annihilates $W_{p} \oplus Y_{p}$ as well. Thus $d H_{p}$ is naturally an element of $Z_{p}^{*} \cong \mathfrak{g}_{\mu} / \mathfrak{g}_{p}$, independent of the choice of representative for $Z_{p}$.

Proposition 2.3 Let $p \in P$ be a relative equilibrium for the Hamiltonian system $H$, and suppose that $X_{H}(p)=X_{\xi}(p)$ (so $\xi$ is well-defined modulo $\mathfrak{g}_{p}$ ). Then, viewing $d H_{p}$ as an element of $Z_{p}^{*} \cong\left(\mathfrak{g}_{\mu} / \mathfrak{g}_{p}\right)$ as described above, we have

$$
d H_{p}=[\xi] \in \mathfrak{g}_{\mu} / \mathfrak{g}_{p} .
$$

Proof The isomorphism $Z_{p}^{*} \cong\left(\mathfrak{g}_{\mu} / \mathfrak{g}_{p}\right)$ is defined via the symplectic form:

$$
[\xi] \in \mathfrak{g}_{\mu} / \mathfrak{g}_{p} \mapsto\left[z \mapsto \omega\left(X_{\xi}(p), z\right)\right] \in Z_{p}^{*} .
$$

Since $X_{H}(p)=X_{\xi}(p)$, it follows that for $z \in Z_{p}, d H_{p}(z)=\omega\left(X_{H}(p), z\right)=\omega\left(X_{\xi}(p), z\right)$ as required.

\section{Reduction to $\alpha=0$.}

It turns out that there is no essential difference between bifurcations of relative equilibria occurring near $\Phi(p)=0$ and near $\Phi(p)=\alpha \neq 0$, provided one replaces $G$ by $G_{\alpha}$. We now show how one 
reduces from the case $\alpha \neq 0$ to the case $\alpha=0$, and in the remainder of the paper we will assume $\alpha=0$.

Following [7] (\$41), let $S_{\alpha}$ be a $G_{\alpha}$-invariant slice to the coadjoint orbit $O_{\alpha}$ at $\alpha=\Phi(p) \in \mathfrak{g}^{*}$. Note that $S_{\alpha}$ can be identified with a neighbourhood of zero in $\mathfrak{g}_{\alpha}^{*}$, since $T_{\alpha} O_{\alpha}=\mathfrak{g}_{\alpha}^{\perp} \subset \mathfrak{g}^{*}$. Thus there is a natural projection of a neighbourhood of $\alpha$ in $\mathfrak{g}^{*}$ on to $S_{\alpha}$.

By equivariance, the image of $d \Phi_{p}$ contains the tangent space to the coadjoint orbit, so that $\Phi$ is transverse to $S_{\alpha}$. Then $Q=Q_{\alpha}=\Phi^{-1}\left(S_{\alpha}\right)$ is (near $p$ ) a symplectic submanifold of $P$ with $T_{p} Q \oplus X_{p}=$ $T_{p} P$ - see [7] (Theorem 26.7). Moreover, $Q$ is $G_{\alpha}$-invariant, and the $Q$-momentum map $\Phi_{Q}$ is just the restriction to $Q$ of $\Phi$, composed with the natural projection onto $S_{\alpha} \subset \mathfrak{g}_{\alpha}^{*}$ :

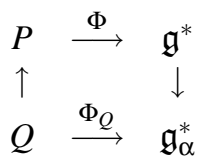

By the local model for $G_{\alpha}$ actions, we have

$$
Q_{\alpha} \stackrel{\sim}{\longrightarrow} G_{\alpha} \times_{G_{p}} S_{p}
$$

where $S_{p}$ is a slice to the $G_{\alpha} . p$ in $Q_{\alpha}$, which is also a slice to $G . p$ in $P$. By the local model for symplectic $G_{\alpha}$ actions, we have moreover

$$
Q_{\alpha} \stackrel{\sim}{\longrightarrow} G_{\alpha} \times_{G_{p}}\left(\mathfrak{m}^{*} \times Y_{p}\right)
$$

Consequently, $P \stackrel{\sim}{\longrightarrow} G \times_{G_{\alpha}} Q_{\alpha}$, see [4] (Chapter II). It follows that $P / G$ and $Q_{\alpha} / G_{\alpha}$ are isomorphic, as are the corresponding reduced spaces.

Since $\alpha$ is in the centre of $\mathfrak{g}_{\alpha}^{*}$, it follows that $\Phi_{Q}^{\prime}:=\Phi_{Q}-\alpha$ is also a momentum map for the $G_{\alpha}$ action on $Q$, with $\Phi_{Q}^{\prime}(p)=0$. In this fashion, we can reduce the general case to one with $\Phi(p)=0$.

There is one useful formula to check, namely that $w\left(\mu ; G_{\alpha}\right)=w(\mu) / w(\alpha)$, where $w\left(\mu ; G_{\alpha}\right)$ is the cardinality of the orbit of $\mu$ under the Weyl group $W_{\alpha}$ of $G_{\alpha}$.

Proposition 2.4 Let $\mu \in S_{\alpha} \cong \mathfrak{g}_{\alpha}^{*}$. Then $w\left(\mu ; G_{\alpha}\right)=w(\mu) / w(\alpha)$.

ProOF Let $T$ be a maximal torus of $G_{\alpha}$. Then it is also a maximal torus of $G$, since every $G_{\alpha}$ contains a maximal torus of $G$. It follows that the normalizers of $T$ satisfy

$$
N\left(T ; G_{\alpha}\right) \subset N(T ; G)
$$

If we quotient by $T$, we obtain

$$
W_{\alpha} \subset W
$$

Identify $\mathfrak{g}_{\alpha}$ and $\mathfrak{g}_{\alpha}^{*}$ by means of an invariant inner product on $\mathfrak{g}_{\alpha}$, and let $\mu \in \mathfrak{g}_{\alpha}$. We can choose $T$ so that $\mu \in \mathfrak{t}$. The result now follows by the slice theorem applied to the Weyl group action:

$$
W . \mu=W \times_{W_{\alpha}} W_{\alpha} \cdot \mu .
$$

From now on, we will assume $\alpha=0$. 


\section{Free actions}

In this section, we improve on Theorem 1.2 in the case that the group acts freely on $P$ (at least in a neighbourhood of $p$ ). As described at the end of the section above, we can suppose without loss of generality that $\Phi(p)=0$. The momentum map $\Phi$ is then a submersion, and the orbit space $P / G$ is a smooth manifold, which contains the Meyer-Marsden-Weinstein reduced spaces $P_{\mu}=\varphi^{-1}\left(O_{\mu}\right)$. Note that for free actions $\mathfrak{g}_{p}=0$, so that Corollary 2.2 gives the local model for a free action as

$$
\mathfrak{g}^{*} \times Y \stackrel{\sim}{\longrightarrow} P / G
$$

(we now suppress the $p$-subscript on $Y$ ) and the orbit momentum map is

$$
\varphi(\mu, y)=O_{\mu} \in \mathfrak{g}^{*} / G
$$

The reduced spaces are thus, in a neighbourhood $U$ of $\bar{p} \in P / G$,

$$
P_{\mu}=O_{\mu} \times Y
$$

And in particular, $P_{0}=Y$. Consequently, in $U$

$$
P_{\mu}=O_{\mu} \times P_{0}
$$

One therefore has a family of Hamiltonian systems parametrized by $\mu \in \mathfrak{g}^{*}$ (or more precisly by $\left.O_{\mu} \in \mathfrak{g}^{*} / G\right)$, and in this family, the phase space can change not only its topology, but even its dimension. This fact is at the heart of the bifurcations of relative equilibria that occur for free actions.

Theorem 3.1 Suppose that the compact group $G$ acts freely on $P$ and that $G$. $p$ is a relative equilibrium in $P$, with $\Phi(p)=0$. Suppose moreover that in the reduced space $P_{0}$ the relative equilibrium is nondegenerate. Then there is a G-invariant neighbourhood $U$ of G.p, and an invariant neighbourhood $V$ of 0 in $\mathfrak{g}^{*}$, such that for each $\mu \in V$, there are at least $\frac{1}{2} \operatorname{dim}\left(O_{\mu}\right)+1$ relative equilibria in $P_{\mu}$, and at least $w(\mu)$ if they are all non-degenerate.

Let $p$ lie on a relative equilibrium where the group acts freely, that is, a point of trivial isotropy. Being a relative equilibrium, we have that there is a (unique) $\xi \in \mathfrak{g}$ satisfying,

$$
X_{\xi}(p)=X_{H}(p)
$$

This $\xi$ is said to be the velocity of the relative equilibrium; it is defined modulo the adjoint action. If, in addition to the action being free, $\xi$ is a regular element of $\mathfrak{g}$, we say that the relative equilibrium is regular (some authors use this adjective differently). Dynamically, this corresponds to the trajectory through $p$ being dense in a maximal torus of the group orbit.

Theorem 3.2 If in addition to the above hypotheses, the relative equilibrium $\bar{p}=G . p \in P_{0}$ is regular, then there are precisely $w(\mu)$ relative equilibria in $P_{\mu}$, all of which are non-degenerate. Moreover, if $\bar{p}$ is formally stable in $P_{0}$ (i.e. $d^{2} H_{0}(\bar{p})$ is definite) then precisely one of these is formally stable (except in the case $\Phi^{-1}(0)=$ G.p where there are two).

The first of these theorems is proved in this section, and the second is proved near the end of $\S 4$. 
Remarks 3.3 (a) Recall that a regular element of a Lie algebra is an element contained in only one Cartan subalgebra. The set of elements of $\mathfrak{g}$ that are not regular is of codimension at least 3 [5, p.190], and so this is a reasonable genericity hypothesis. In [14] we consider in greater detail the case where the velocity of the relative equilibrium is not regular.

(b) If in either theorem, $d^{2} H_{0}(\bar{p})$ is positive definite, then the relative equilibrium on $P_{\mu}$ with least energy will be (formally) stable.

(c) For classical mechanical systems, with $H(p, q)=K(p)+V(q)$, the momentum map is given by $\langle\Phi(p, q), \xi\rangle=\left\langle p, X_{\xi}(q)\right\rangle$. Since the kinetic energy $K$ is positive definite, it follows that the only relative equilibria on $\Phi^{-1}(0)$ have zero velocity, so are not regular. Consequently, in classical mechanical systems, Theorem 3.2 will not apply directly, but only via Proposition 2.4, whence it follows that if $\Phi(p)=\alpha \neq 0$, then in a neighbourhood of $\bar{p}$ there are $w(\mu) / w(\alpha)$ relative equilibria on $P_{\mu}$. See the example below (where $w(\alpha)=1$ ).

(d) The estimate $\frac{1}{2} \operatorname{dim}\left(O_{\mu}\right)+1$ for the number of relative equilibria in Theorem 3.1 is a lower bound for the Ljusternik-Shnirelman category of the coadjoint orbit $O_{\mu}$ arising from its cup-length (see the end of this section).

(e) Under the same hypotheses as Theorem 3.2, Patrick [18] shows that the set of relative equilibria forms a smooth submanifold of phase space of dimension $\operatorname{dim} G+\operatorname{rank} G$. Clearly, $G$ acts freely on this manifold, so its image in $P / G$ is a submanifold of dimension $\operatorname{rank} G$.

Example 3.4 In his thesis [16], Patrick gives an example of a regular relative equilibrium. It consists of two identical axisymmetric rigid bodies, coupled by an ideal ball and socket joint. The symmetry group is $G=\mathbf{S O}(3) \times S^{1} \times S^{1}$, the configuration space is $Q=\mathbf{S O}(3) \times \mathbf{S O}(3)$ and the phase space its cotangent bundle $P=T^{*} Q$. For $\alpha=\left(0, \mu_{1}, \mu_{2}\right) \in \mathfrak{g}^{*}=\mathfrak{s o}(3)^{*} \times \mathbf{R} \times \mathbf{R}$, with $\mu_{1} \neq \pm \mu_{2}$, Patrick describes a relative equilibrium consisting of the two bodies in a ' $\mathrm{V}$ '-shape, with the ' $\mathrm{V}$ ' rotating about an axis in the plane of the ' $\mathrm{V}$ ' in one direction, and the two bodies rotating about their axes (the arms of the ' $V$ ') in the opposite sense, so that the total angular momentum is zero (see also [18, Figure 2]). The momentum value $\alpha$ is not regular, indeed $\mathfrak{g}_{\alpha}=\mathfrak{g}$, but the velocity is a regular element of $\mathfrak{g}$. Patrick shows that this relative equilibrium is (formally) stable.

Consider now $\mu=\left(v, \mu_{1}, \mu_{2}\right) \in \mathfrak{g}^{*}$, with $v$ small. According to Theorem 3.2 there are two relative equilibria on $P_{v}$ (close to the given one), one of which is stable, and the other is not (or at least, $d^{2} H_{\mu}$ is not definite there). These correspond to spinning the ' $\mathrm{V}$ ' faster and slower about its axis (respectively), by an amount depending on $|v|$, leaving the spins of each body unchanged. Moreover, it is the one with less energy which is stable; that is, the one that spins slower. These conclusions are indeed borne out by Patrick's work.

Let $H: P \rightarrow \mathbf{R}$ be a smooth $G$-invariant Hamiltonian. Then $H$ defines a smooth function $\bar{H}$ : $P / G \rightarrow \mathbf{R}$, whose restriction to the reduced space $P_{\mu}$ we denote by $H_{\mu}$. We consider $\bar{H}$ as a function on $P_{0} \times \mathfrak{g}^{*}$, and $H_{\mu}$ its restriction to the symplectic space $P_{0} \times O_{\mu}$ (via the isomorphism of Corollary 2.2).

A point $p \in P$ lies on a relative equilibrium if $X_{H}(p) \in \mathfrak{g} \cdot p$, or equivalently if $d H_{\alpha}(\bar{p})=0$, where $\Phi(p)=\alpha$, and $\bar{p}$ is the group orbit containing $p$. The non-degeneracy of the relative equilibrium corresponds to the quadratic form $d^{2} H_{\alpha}$ being non-degenerate.

Proposition 3.5 If $\bar{p} \in P / G$ is a non-degenerate relative equilibrium with momentum 0 , then there is a diffeomorphism $\gamma$ of a neighbourhood of $\bar{p}$ in $P / G=Y \times \mathfrak{g}^{*}$ of the form

$$
\gamma(y, \mu)=\left(\gamma_{1}(y, \mu), \mu\right)
$$

such that

$$
\bar{H} \circ \gamma(y, \mu)=Q(y)+h(\mu) .
$$


Here $Q$ is the non-degenerate quadratic form $Q=\frac{1}{2} d^{2} H_{0}(\bar{p})$, and $h$ is a smooth function on $\mathfrak{g}^{*}$.

ProOF This is just the splitting lemma, see for example [19].

Note that the fact that $\gamma$ satisfies $\gamma(y, \mu)=\left(\gamma_{1}(y, \mu), \mu\right)$ means that the diffeomorphism preserves the "cylinders" $P_{\mu}=Y \times O_{\mu}$. Consequently, for $\nu \in O_{\mu}$,

$$
H_{\mu}(y, v)=Q(y)+h_{\mu}(v)
$$

where $h_{\mu}$ is the restriction of $h$ to $O_{\mu}$. With these coordinates, then, $H_{\mu}$ has a critical point at $(y, \mu)$ iff $y=0$ and $h_{\mu}$ has a critical point at $\mu$, with moreover

$$
\operatorname{ind}\left(H_{\mu}\right)=\operatorname{ind}(Q)+\operatorname{ind}\left(h_{\mu}\right),
$$

where ind is the Morse index of a critical point. Thus the relative equilibria near $\bar{p}$ are just the critical points of $h_{\mu}$ as $\mu$ varies near $\alpha$. It should perhaps be pointed out that $\gamma$ will not preserve the Poisson structure, otherwise the dynamics would decouple!

We have thus reduced the problem of finding relative equilibria near $\bar{p} \in P / G$ to a problem of finding critical points of a function $h$ on each nearby coadjoint orbit, and are now in a position to prove Theorem 3.1.

Proof (of Theorem 3.1) We have shown that relative equilibria with momentum $\mu$ are just the critical points of a function $h_{\mu}: O_{\mu} \rightarrow \mathbf{R}$.

Given any smooth function on a compact manifold $M$, the Ljusternik-Shnirelman category cat $(M)$ of $M$ provides a lower bound for the number of critical points of the function. A standard result [6] states that the Ljusternik-Shnirelman category of a compact manifold is at least 1 greater than the cup-length of the manifold (in general this is a rather weak estimate), and for any simply connected compact symplectic manifold (such as a coadjoint orbit)

$$
\operatorname{cup} \text {-length }(M)=\operatorname{dim}(M) / 2 \text {. }
$$

This follows since $\omega^{n}$ is a nowhere vanishing multiple of the volume form (where $\operatorname{dim}(M)=2 n$, and $\omega$ is the symplectic form), and hence by Stokes' theorem $[\omega]^{n}=\left[\omega^{n}\right] \neq 0$ in the top cohomology group of the manifold.

It is well-known (by Morse-theoretic arguments) that any smooth function on $O_{\mu}$ with only nondegenerate critical points has at least $w(\mu)$ critical points [3]. Moreover, if the relative equilibrium on $P_{0}$ is formally stable, then a corresponding extremum of $h_{\mu}$ (max or min corresponding to max or min of $H_{0}$ ) gives rise to a formally stable equilibrium point on $P_{\mu}$.

Remark 3.6 If the action is locally free rather than free, so that $G_{p}$ is finite, and $\mathfrak{g}_{p}=0$, then if $S$ is the slice at $p$, we have $P / G \cong S / G_{p}$. In terms of the symplectic decomposition,

$$
P / G \cong\left(\mathfrak{g}^{*} \times P_{0}\right) / G_{p}
$$

If $H: P / G \rightarrow \mathbf{R}$ is a Hamiltonian defined in a neighbourhood of $p$, then it can be lifted to a $G_{p}$ invariant function on the slice $\mathfrak{g}^{*} \times P_{0}$. If the restriction of this invariant function to $P_{0}$ is non-degenerate, then the same reduction procedure can be applied as above, to obtain a $G_{p}$ invariant function $h$ on $\mathfrak{g}^{*}$. Again, one is interested in the critical points of the restriction of this $h$ to the coadjoint orbits in $\mathfrak{g}^{*}$. However, the difference now, is that distinct critical points might not correspond to distinct relative equilibria. It would be necessary to know how $G_{p}$ acts on the set of critical points: two critical points being in the same $G_{p}$-orbit if and only if they correspond to the same relative equilibrium. These ideas are elaborated in [14]. 


\section{Critical points of linear functions on coadjoint orbits}

As usual, $G$ is a compact Lie group, $\mathfrak{g}$ its Lie algebra, and $\mathfrak{g}^{*}$ the dual of the Lie algebra. The group $G$ acts naturally on $\mathfrak{g}$ and $\mathfrak{g}^{*}$ by its adjoint and coadjoint actions respectively. Since $G$ is compact, there is an invariant metric on $\mathfrak{g}$, and consequently these two actions are isomorphic. We denote by $W$ the Weyl group of $G$, which acts on the Lie algebra $\mathfrak{t}$ of any maximal torus, and on its dual $\mathfrak{t}^{*}$. The orbit of a point $\mu \in \mathfrak{t}^{*}$ is denoted by $W(\mu)$, and the number of points in that orbit by $w(\mu)$.

Let $\xi \in \mathfrak{g}$ and $\mu \in \mathfrak{g}^{*}$. Then $\xi$ defines a linear function on $\mathfrak{g}^{*}$ which restricts to a function,

$$
\xi_{\mu}: O_{\mu} \rightarrow \mathbf{R}
$$

We are interested in the critical points of such functions.

Proposition 4.1 If we identify $\mathfrak{g}^{*}$ with $\mathfrak{g}$ via a G-invariant inner product, then the singular locus of the function $\xi_{\mu}$ is given by

$$
\Sigma\left(\xi_{\mu}\right)=\mathfrak{g}_{\xi} \cap O_{\mu}
$$

PROOF The function $\xi_{\mu}$ is the Hamiltonian function for the vector field $\nu \mapsto \operatorname{ad}_{\xi} \nu$ on $O_{\mu}$. Critical points of $\xi_{\mu}$ thus correspond to zeros of the vector field $\operatorname{ad}_{\xi} v$, and this vector field has a zero at $v$ if and only if $\xi \in \mathfrak{g}_{\mathrm{v}}$.

The remainder is clear: if we write $\hat{\mu}$ for the image in $\mathfrak{g}$ of $\mu \in \mathfrak{g}^{*}$ under the identification, then $\xi \in \mathfrak{g}_{\mu}$ if and only if $\hat{\mu} \in \mathfrak{g}_{\xi}$ (iff $[\xi, \hat{\mu}]=0$ ).

Recall that $\xi \in \mathfrak{g}$ is regular if it is contained in a unique Cartan subalgebra. If one considers a fixed Cartan subalgebra $\mathfrak{t}$ of $\mathfrak{g}$, the regular elements are those points not contained in a reflexion hyperplane for the Weyl group action. The set of non-regular (or singular) elements of $\mathfrak{g}$ is a set of codimension at least 3 , see [5].

Proposition 4.2 Let $\xi$ be a regular element of $\mathfrak{g}$, and $\mu \in \mathfrak{g}^{*}$. Then the function $\xi_{\mu}: O_{\mu} \rightarrow \mathbf{R}$ has precisely $w(\mu)$ critical points, all of which are non-degenerate.

PROOF Let $\mathfrak{t}$ be the unique Cartan subalgebra in $\mathfrak{g}$ containing $\xi$; that is, $\mathfrak{t}=\mathfrak{g}_{\xi}$. We have that $\Sigma\left(\xi_{\mu}\right)=\mathfrak{t} \cap O_{\mu}$, and by standard results on Weyl groups, this is precisely the Weyl group orbit $W . \mu$.

The fact that these critical points are non-degenerate follows from the fact that critical points of momentum maps for linear actions are homogeneous quadratic (in appropriate local coordinates), so a critical point is non-degenerate if and only if it is an isolated critical point. The linear action here is that of $T(\xi)$ on $\mathfrak{g}^{*}$, where $T(\xi)$ is the maximal torus generated by $\xi$.

There is a well-known setting for visualizing the critical points of these linear functions on coadjoint orbits, which is as follows. As in the preceding proof, let $\mathfrak{t}$ be the unique Cartan subalgebra containing $\xi$, which is the Lie algebra of the maximal torus $T=T(\xi)$. The function (projection) $\xi: \mathfrak{g}^{*} \rightarrow \mathbf{R}$ can be factored via the projection $\pi: \mathfrak{g}^{*} \rightarrow \mathfrak{t}^{*}$, followed by $\xi_{\mathfrak{t}^{*}}: \mathfrak{t}^{*} \rightarrow \mathbf{R}$. The restriction to $O_{\mu}$ of the projection $\pi$ is in fact the momentum map for the $T$ action on $O_{\mu}$. A few examples are shown in Figure 2.

The image of a coadjoint orbit under the projection to $t^{*}$ is a convex polytope (a result due to Kostant [8], see also [3]). The map $\pi_{O_{\mu}}$ has rank 0 at each vertex (they correspond to the fixed points for the action of $T$ ), and these are the only points of rank 0 . Consequently, $\xi_{\mu}=\xi_{O_{\mu}}$ will also have rank 0 at these points; that is they will be critical points. These vertices correspond to points of intersection 


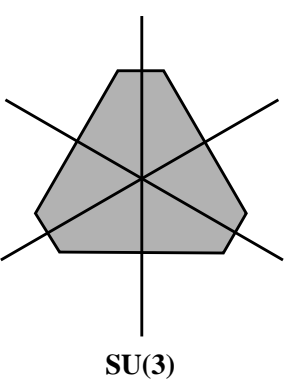

$\mu$ regular

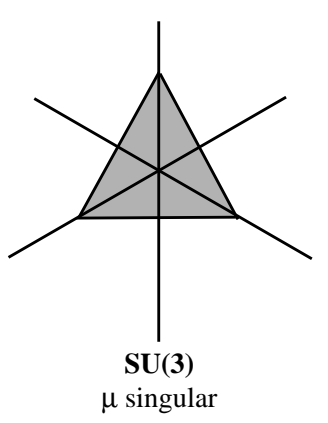

singular

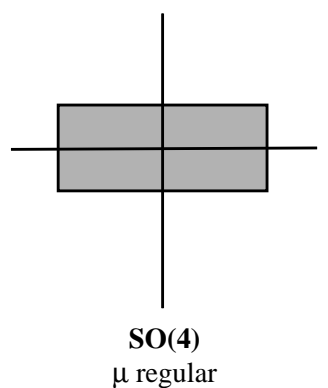

$\mu$ regular

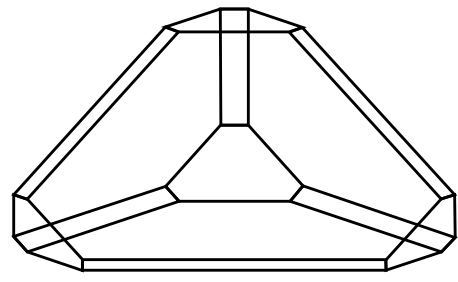

SU(4)

$\mu$ regular

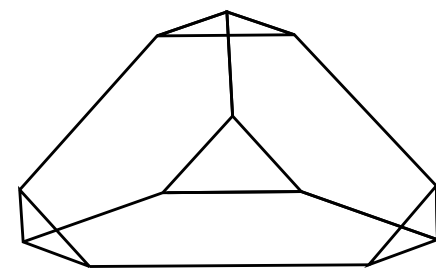

SU(4)

$\mu$ singular

Figure 2: Projections of some coadjoint orbits to $\mathfrak{t}^{*}$ : see text

$O_{\mu} \cap \mathfrak{t}^{*}$ (see Proposition 4.1), and so form a Weyl group orbit. Hence the convex polytope is also invariant under the Weyl group.

The $\xi \in \mathfrak{t}$ that are regular are precisely those that are not orthogonal to any of the faces in the convex polytope, and it follows that the composition $\xi \circ \pi_{\rho_{\mu}}$ has no other critical points. (A singular $\xi \in \mathfrak{t}$ would have infinitely many critical points on $O_{\mu}$, and the argument below showing that for regular $\xi$, the higher order terms in $h=\xi+\cdots$ can be removed, would no longer hold.)

In Figure 2, the first two diagrams are for $G=\mathbf{S U}(3)$, where $W=S_{3}$ (the symmetric group on 3 letters). If we take $\mu$ to be generic (i.e. regular), then the polytope is a 'semiregular' hexagon, and $\operatorname{dim}\left(O_{\mu}\right)=6$; while if we take $\mu$ in one of the lines of reflexion, the polytope degenerates to an equilateral triangle and $\operatorname{dim}\left(O_{\mu}\right)=4$ (of course, if $\mu=0$, then $O_{\mu}=0$ and the polytope further degenerates to a point). Each of the vertices corresponds to a critical point of any generic linear function on $\mathfrak{t}=\mathbf{R}^{2}$. The third diagram is for $G=\mathbf{S O}(4)$ and also for $G=\mathbf{S O}(3) \times \mathbf{S O}(3)$ (these have the same Weyl group, $W=\mathbf{Z}_{2} \times \mathbf{Z}_{2}$ ). The two line diagrams are for $G=\mathbf{S U}(4)$, with $W=S_{4}$, which has order 24 (and acts on $\mathfrak{t}^{*}=\mathbf{R}^{3}$ as the standard symmetry group of the tetrahedron). A typical polyhedron for regular $\mu$ is shown on the left (here $\operatorname{dim}\left(O_{\mu}\right)=12$ ), while a particular degeneration $(\mu$ is fixed by one of the reflexions in the tetrahedral group, giving a truncated tetrahedron) is shown on the right (here $\operatorname{dim}\left(O_{\mu}\right)=10$ ). The other degenerations are an octahedron (with $\operatorname{dim}\left(O_{\mu}\right)=8$ ) and a tetrahedron (with $\operatorname{dim}\left(O_{\mu}\right)=6$ ) according to the isotropy of $\mu$. For $\mathbf{S O}(7)$, which has rank 3, the Weyl group is the group of symmetries of the cube. Regular $\mu$ gives rise to a polytope with 48 vertices (with $\operatorname{dim}\left(O_{\mu}\right)=18$ ), and it is a nice exercise to visualize all the possible degenerations (five, excluding 
$\mu=0)$.

Now suppose that the function $h: \mathfrak{g}^{*} \rightarrow \mathbf{R}$ in which we are interested is not linear.

Proposition 4.3 If $h: \mathfrak{g}^{*} \rightarrow \mathbf{R}$ is a smooth function such that $d h_{0}=\xi \in \mathfrak{g}$ is a regular element of $\mathfrak{g}$, then for all $\mu \in \mathfrak{g}^{*}$ sufficiently small, there are $w(\mu)$ critical points of the restriction $h_{\mu}=h_{O_{\mu}}$, all of which are non-degenerate.

Proof We use an argument involving blowing-up $\mathfrak{g}^{*}$ at 0 to show that for $\mu$ sufficiently small, the critical points of $h_{\mu}$ are in 1-1 correspondence with critical points of $\xi_{\mu}$. The result then follows.

Let $S$ be the unit sphere in $\mathfrak{g}^{*}$, with respect to some $G$-invariant norm. Consider the blowing-up

$$
\begin{aligned}
& \mathbf{R} \times S \longrightarrow \mathfrak{g}^{*} \\
& (r, \theta) \mapsto r \theta .
\end{aligned}
$$

Write $h(r, \theta)$ rather than $h(r \theta)$. Without loss of generality, we can assume $h(0, \theta)=0$, since we are only interested in critical points of $h$. Then,

$$
h(r, \theta)=r h_{1}(r, \theta)
$$

for some smooth function $h_{1}$. Note that since coadjoint orbits lie in spheres, the critical points of the restrictions of $h$ and $h_{1}$ to coadjoint orbits coincide. By Taylor's theorem we have

$$
h_{1}(r, \theta)=\langle\xi, \theta\rangle+r R(r, \theta) \text {. }
$$

Now, if $\xi \in \mathfrak{g}$ is a regular element, then its restriction (as an element of $\left(\mathfrak{g}^{*}\right)^{*}$ ) to any coadjoint orbit has only non-degenerate critical points, and precisely $w(\mu)$ such critical points on the coadjoint orbit through $\mu \in S$. Using the implicit function theorem and the compactness of coadjoint orbits, we conclude that for $r$ sufficiently small, $h_{1}$ has precisely $w(\mu)$ critical points on the coadjoint orbit through $r \mu$ in $r S$, all of which are non-degenerate.

PROOF (of Theorem 3.2) We now return to the setting of \$3. Starting from an invariant Hamiltonian $H: P \rightarrow \mathbf{R}$, we used the local model (3.1) and the splitting lemma (Proposition 3.5) to reduce the problem of finding relative equilibria of $H$ (near a given one) to finding critical points of the restrictions to the coadjoint orbits of a function $h: \mathfrak{g}^{*} \rightarrow \mathbf{R}$. By Proposition 2.3, we have that $d H_{p}=d h_{p}=\xi$, where $X_{\xi}(p)=X_{H}(p)$ is the velocity at $p$ of the relative equilibrium. By hypothesis, we assume that $\xi \in \mathfrak{g}$ is regular, so that we can apply Proposition 4.3, to obtain the existence result.

For the stability, suppose that $d^{2} H_{0}(p)$ is positive definite, then of the nearby relative equilibria, only those for which $h_{\mu}$ has a local minimum are formally stable. But $\xi_{\mu}$ (for $\xi$ regular) has a unique minimum [3], and by the blow-up argument of Propsotion 4.3 the same holds for $h_{\mu}$, so we are done. (If $\Phi^{-1}(0)=G . p$, then $P_{0}$ is a point, so both the maximum and the minimum of $h_{\mu}$ will be formally stable, as happens for the rigid body.)

We conclude by briefly considering the eigenvalues of the bifurcating relative equilibria. Recall that an equilibrium point of a linear Hamiltonian system $\dot{z}=L z$ on the symplectic space $V$ is strongly stable (also called parametrically stable) if every nearby linear system is spectrally stable (has only imaginary eigenvalues). 
Suppose that the linearization $L_{0}$ of the regular relative equilibrium $\bar{p} \in P_{0}$ is strongly stable. Then for $\mu$ close to 0 , the $w(\mu)$ relative equilibria on $P_{\mu}$ will have eigenvalues close to those of $L_{0}$, together with new eigenvalues corresponding to the $\xi$-action on $O_{\mu}$.

By hypothesis, the former are all imaginary (even after perturbation to $\mu$ ). For geometric reasons, the latter are also imaginary. Indeed, the action of a regular element $\xi \in \mathfrak{g}$ on $O_{\mu}$ is the restriction of the infinitesimal coadjoint action on $\mathfrak{g}^{*}$, and this action, like the adjoint action to which it is isomorphic, is such that all the non-zero eigenvalues are imaginary and distinct; they can be obtained from the infinitesimal roots of the Lie group, see for example Chapter V of [5]. Denote by $\mathcal{R}$ the set of these infinitesimal roots, which we can view as lying in $\mathfrak{t}^{*}$, where $\mathfrak{t}$ is the unique Cartan subalgebra containing $\xi$. The eigenvalues of $\operatorname{ad}_{\xi}$ are then

$$
\mathcal{R}(\xi):=\{i\langle r, \xi\rangle \mid r \in \mathcal{R}\},
$$

where $i=\sqrt{-1}$. Note that $0 \notin \mathcal{R}(\xi)$, since the zero eigenspace is precisely the maximal torus containing $\xi$, which is orthogonal to the tangent space to $O_{\mu}$ at a critical point of $\xi_{\mu}$ (by Proposition 4.1). Since $\xi$ is regular, it also follows that all the eigenvalues of ad $\xi$ are simple. Consequently, the equilibria of the vector field $a d_{\xi}^{*}$ on $O_{\mu}$ are strongly stable. It follows that the equilibria of $h_{\mu}$ are also strongly stable.

One would therefore expect the relative equilibria on $P_{\mu}$ to be strongly stable. However, it may happen that an eigenvalue of $L_{0}$ coincides with an eigenvalue of ad $\xi$; it would then appear likely that in the perturbation to $\mu \neq 0$ there could be a "Hamiltonian-Hopf" (or "complex instability") bifurcation. It is reasonable to suppose that such coincidence of eigenvalues between $L_{0}$ and ad $\xi$ is non-generic, of codimension 1.

\section{References}

[1] J.M. Arms, A. Fischer, J.E. Marsden, Une aproche symplectique pour des théorèmes de décomposition en géométrie ou relativité générale. C. R. Acad. Sci. Paris 281 (1975), 517-520.

[2] V.I. Arnold, Mathematical Methods of Classical Mechanics. Springer-Verlag, 1978.

[3] M.F. Atiyah, Convexity and commuting Hamiltonians. Bull. L.M.S. 14 (1982), 1-15.

[4] G.E. Bredon, Introduction to Compact Transformation Groups. Academic Press, Orlando, 1972.

[5] Th. Bröcker and T. tom Dieck, Representations of Compact Lie Groups. Springer-Verlag, New York, 1985.

[6] B.A. Dubrovin, A.T. Fomenko and S.P. Novikov, Modern geometry, methods and applications, Part 2: The geometry and topology of manifolds. Springer-Verlag, New York, 1985.

[7] V. Guillemin and S. Sternberg, Symplectic Techniques in Physics. Cambridge University Press, Cambridge, 1984.

[8] B. Kostant, On convexity, the Weyl group and the Iwasawa decomposition. Ann. Scien. Ec. Norm. Sup. 6 (1973), 413-455.

[9] P. Libermann and C.-M. Marle, Symplectic Geometry and Analytical Mechanics. D. Reidel Publishing Company, Dordrecht, 1987.

[10] C.-M. Marle, Modèle d'action hamiltonienne d'un groupe de Lie sur une variété symplectique. Rend. Sem. Mat. Univers. Politecn. Torino 43 (1985), 227-251.

[11] J. Marsden, Lectures on Mechanics. LMS Lecture Notes 174, CUP, Cambridge, 1992.

[12] J. Marsden and A. Weinstein, Reduction of symplectic manifolds with symmetry. Rep. Math. Phys 5 (1974), $121-130$. 
[13] K. Meyer, Symmetries and integrals in mechanics. In Dynamical Systems (M. Peixoto, ed.), 259-273. Academic Press, New York, 1973.

[14] J. Montaldi and R.M. Roberts, Relative equilibria of molecules. (In preparation)

[15] J. Montaldi, R.M. Roberts, and I. Stewart, Periodic solutions near equilibria of symmetric Hamiltonian systems. Phil. Trans. Roy. Soc. London 325 (1988), 237-293.

[16] G. Patrick, Two axially symmetric coupled rigid bodies: relative equilibria, stability, bifurcations, and a momentum preserving symplectic integrator. Ph.D. Thesis, Department of Mathematics, University of California, Berkeley, May 1991.

[17] G. Patrick, Relative equilibria in Hamiltonian systems: The dynamic interpretation of nonlinear stability on a reduced phase space. J. Geom. Phys. 9 (1992), 111-119.

[18] G. Patrick, Relative equilibria of Hamiltonian systems with symmetry: linearization, smoothness and drift. J. Nonlinear Sci. 5 (1995), 373-418.

[19] T. Poston and I. Stewart, Catastrophe Theory and its Applications. Pitman, San Francisco, 1978.

[20] R.M. Roberts and M.E.R. de Sousa Dias, Bifurcations from relative equilibria of Hamiltonian systems. University of Warwick preprint (1996).

[21] R. Sjamaar and E. Lerman, Stratified symplectic spaces and reduction. Annals of Math. 134 (1991), 375422.

[22] A. Weinstein, Stability of Poisson-Hamilton equilibria. Cont. Math. A.M.S. 28 (1984), 3-14. 